\title{
Dual-mode-locking mechanism for an akinetic dispersive ring cavity swept source
}

\author{
Radu F. Stancu* and Adrian Gh. Podoleanu \\ Applied Optics Group, University of Kent, School of Physical Sciences, Canterbury, Kent, CT2 7NH, UK \\ *Corresponding author: rs478@kent.ac.uk
}

Received November 26, 2014; revised February 22, 2015; accepted February 24, 2015;

posted February 26, 2015 (Doc. ID 228579); published March 23, 2015

\begin{abstract}
A fast dual-mode-locked akinetic optical swept source in the 1550-nm wavelength band is presented that is tested up to a sweep rate of $797 \mathrm{KHz}$. It comprises a voltage-controlled oscillator-driven wideband semiconductor optical amplifier (SOA) along with a dispersion compensation fiber, in a ring laser configuration. A Faraday rotating mirror is employed in the cavity as a reflective element in order to achieve better polarization control. By driving the SOA at a high-MHz-frequency value multiple of the resonant frequency $f_{R}$, equal to the inverse round trip time, a first-mode locking mechanism is imposed. A second locking mechanism consists in sweeping the radio frequency of the locking signal at a rate slightly detuned from $f_{R}$. A dynamic linewidth of $0.8 \mathrm{~nm}$ is assessed by measuring the decay of interference signal strength versus optical path difference in a Mach-Zehnder interferometer. (c) 2015 Optical Society of America

OCIS codes: (230.2035) Dispersion compensation devices; (250.5980) Semiconductor optical amplifiers; (140.3560)

Lasers, ring; (140.4050) Mode-locked lasers.

http://dx.doi.org/10.1364/OL.40.001322
\end{abstract}

Wavelength-tunable lasers represent a modern and versatile solution for telecom, sensing, and optical-coherence tomography (OCT) applications, therefore several principles of swept sources (SS) have been reported so far. The most successful design in terms of tuning speed and bandwidth rely on mechanical means such as polygon mirrors [1] or tunable Fabry-Perot filters (TFPF) [2]. Modern Fabry-Perot filters use micro-electro-mechanical systems (MEMS) that have fixed scanning range [3]. Recently, the interest on akinetic principles has been revived by the demonstration of fast, wide tuning bandwidth and large coherence-length SS based on vertical cavity surface emitting laser (VCSEL) devices [4].

A Fourier domain mode-locking (FDML) method was proposed for a TFPF-based ring laser by extending its delay to values matching its round trip time $=1 / f_{R}$, and driving the TFPF at multiples of $f_{R}$. In this way, the limitation imposed by the photon lifetime is circumvented, and $\mathrm{MHz}$ sweep rates are achieved [5].

Yamashita et al. [6,7] proposed an akinetic solution using mode locking in a dispersive cavity, equipped with a semiconductor optical amplifier (SOA) as gain medium and a dispersion compensating fiber (DCF) and achieved hundreds of kHz sweep rates. Later, Takubo and Yamashita expanded the concept by using a reflective SOA and a chirped fiber Bragg grating as dispersive medium [8]. However, the increase in speed was accompanied by significant power reduction. They suggested shortening the cavity length to obtain higher tuning rates compatible with higher output power.

In this Letter, we present a configuration of an akinetic optical swept source (AKSS), which implements a dual locking scheme (DML). A first locking condition is imposed by driving the optical gain at a high frequency, to induce mode locking. A second locking mechanism, inspired from the practice of FDML applied to TFPF lasers [5], employs sweeping at a rate close to $f_{R}$, however in opposition to [5], at essentially different values than $f_{R}$. To produce FDML, the roundtrip time needs to be increased to amenable values, which requires several hundreds of meters of fiber, i.e., this strategy is opposed to that suggested in [8].

The configuration of the AKSS experimented is shown in Fig. 1. It uses a SOA, model SOA-L-C-14-FCA (CIP 1, from $\mathrm{BT}$ ), operating at $1550 \mathrm{~nm}$, maximum current $500 \mathrm{~mA}$, and $3 \mathrm{~dB}$ bandwidth of $50 \mathrm{~nm}$. The SOA is driven by a Thorlabs LDC $210 \mathrm{C}$ power supply. Temperature stability is ensured through thermo-electric cooling provided by a Thorlabs TEC 200C driver (sensor TH $20 \mathrm{k}$ $\Omega$ ). The SOA is delimited by two polarization-insensitive isolators I, which ensure unidirectional lasing. The ring cavity also comprises a circulator, CIR, that directs light through a 110-m length $L_{\mathrm{DCF}}$ of Draka Elite DDU1171023 $\mathrm{DCF}$, with an anomalous dispersion parameter of $-140 \mathrm{ps} / \mathrm{nm} \cdot \mathrm{km}$ at $1550 \mathrm{~nm}$, terminated with a broadband Faraday rotating mirror (FRM), AFW, 60-nm bandwidth at $1550 \mathrm{~nm}$. The total ring cavity length is approximately $255 \mathrm{~m}$.

Output power is drawn out through a 50/50 coupler and represents half of the light in the cavity further amplified by a similar type of SOA. The laser output characteristics of the swept source configuration are measured using an Agilent 86145B optical spectrum analyzer (OSA), with a resolution of $60 \mathrm{pm}$. The coherence length

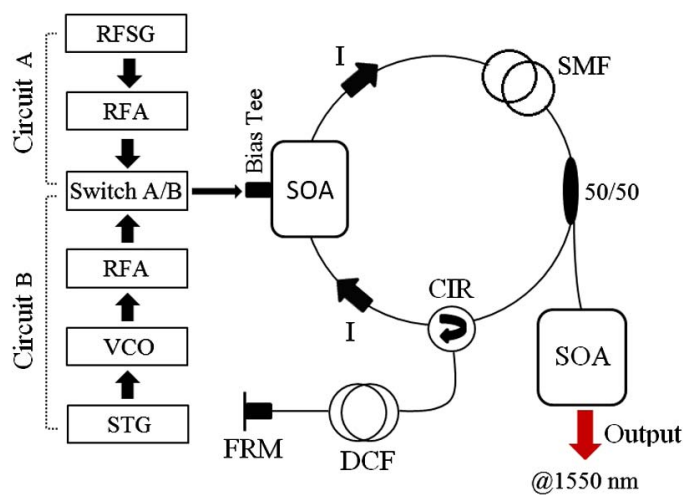

Fig. 1. AKSS configuration. 
of the source is evaluated by driving a Mach-Zehnder interferometer, terminated on a 50/50 coupler ending on a New Focus balanced detector model 1817, DC$80 \mathrm{MHz}, 900-1700 \mathrm{~nm}$ bandwidth, whose output is displayed with a LeCroy Wave Runner 104 MXi-A $1 \mathrm{GHz}$ oscilloscope, with a sampling rate of $10 \mathrm{GS} / \mathrm{s}$ (Gyga sample points per second).

A switch with two positions, A and B, is used to drive the SOA in two regimes of operation. In position A, output from an HP8648C radio-frequency-signal generator (RFSG)-swept synthesizer is used. In this regime, the excitation of signal frequency delivered by the RF synthesizer is varied, to evaluate the static performance of the $\mathrm{SS}$, in terms of its linewidth and tuning bandwidth.

In position $\mathrm{B}$ of the switch, the DML RF mechanism is employed. A low-noise voltage-controlled oscillator (VCO, Mini Circuits ZX-928CA+) and a ZHL-42 W Mini Circuits radio-frequency amplifier, $26 \mathrm{dBm}$ (RFA) are used to drive the SOA with a $5-\mathrm{V}$ amplitude sinusoidal signal applied to the SOA in series with a 27 -ohm resistor. The Agilent 81160A saw-tooth signal (STG) generator provides a saw-tooth signal with adjustable amplitude allowing a versatile control of the tuning bandwidth and of the central frequency of the VCO. In either positions of the switch, the RF signal is injected via a bias tee.

A first principle involved is that of active mode locking of a dispersive ring laser cavity. Dispersion is introduced by the DCF length. The rest of the ring is made of singlemode fiber (SMF, Corning 28e(R), dispersion parameter of $\leq 18 \mathrm{ps} / \mathrm{nm} / \mathrm{km}$ at $1550 \mathrm{~nm})$. Due to dispersion, the index of refraction depends on the optical frequency, and therefore, $f_{R}$ is different for different optical frequencies. By changing the frequency of the signal modulating the gain in the cavity, the optical frequency can be swept. The resonance frequency of the ring laser cavity is defined as

$$
f_{R}=\frac{c}{n L},
$$

where $c$ represents the speed of light in vacuum, $n$ is the refractive index of the cavity, and $L$ is the laser cavity length. The chromatic dispersion in the cavity can be defined for $n$ as a function of wavelength $\lambda$. When a signal of frequency $f_{m}$ modulates the gain in a dispersive cavity, mode locking is induced at the inverse of the roundtrip of light in the cavity. The wavelength tuning range $\Delta \lambda$ is expressed as

$$
\Delta \lambda=-\frac{n_{0}}{c D f_{m_{0}}} \Delta f_{m}
$$

where $f_{m_{0}}$ is the central mode locking frequency, $n_{0}$ is the refractive index at the central optical frequency emitted, $D$ is the dispersion parameter of the ring, and $\Delta f_{m}$ represents the change in the modulation frequency. Maximum tuning range, $\Delta \lambda_{\max }$, is given by:

$$
\Delta \lambda_{\max }=\frac{1}{|D| L f_{m_{0}}} .
$$

The total cavity length determines a total of -30 ps/nm anomalous dispersion in the system. First, the optical linewidth $\delta \lambda$ and tuning bandwidth $\Delta \lambda$ were evaluated

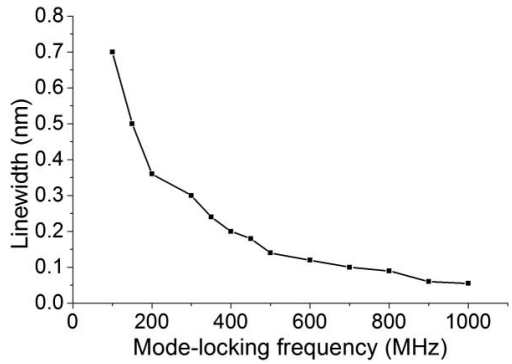

Fig. 2. Linewidth versus mode-locking frequency in the static regime (A).

by switching the switch to position A and operating in static regime, by manually changing the input $\mathrm{RF}$ frequency of the SOA driving signal. The best linewidth of $60 \mathrm{pm}$ was achieved for mode-locking frequencies above $800 \mathrm{MHz}$, as shown by the graph depicted in Fig. 2 . This value matches the resolution of the OSA used, of $60 \mathrm{pm}$, meaning that the linewidth is better than the one measured.

After that, the system is switched to the position B. By changing the dc value of the signal delivered by the pulse generator, the central frequency $f_{m}$ of the VCO is tuned statically. Sweeping rates achieved are no more than $100 \mathrm{kHz}$. The $3-\mathrm{dB}$ bandwidth achieved at $1 \mathrm{kHz}$ is approximately $60 \mathrm{~nm}$. The decay of the optical output as the sweeping speed increases is illustrated in Fig. 3, and it can be noticed that by using the first mode locking mechanism alone, it is not possible to achieve high sweeping rates. Even with short lengths of the cavity, of a couple of meters, we could not achieve sweeping rates larger than $100 \mathrm{kHz}$, as predicted by [8], i.e., the decay of power with the sweeping rate is not due to the long length of fiber used.

Initially, the cavity length is adjusted to a value which determines $f_{R}=782 \mathrm{kHz}$, and the VCO is driven with a signal of frequency $f_{m}$ in the range 360-420 MHz. When the signal applied from the synthesizer to the VCO is swept at a sweeping rate of $f_{S}=f_{R}$, then the VCO spectrum consists in a comb of frequencies $f_{m} \pm f_{R}$, $f_{m} \pm 2 f_{R}, f_{m} \pm 3 f_{R}$, and so on, i.e., in $782-\mathrm{kHz}$ steps. The resulting $\mathrm{RF}$ signal from the $\mathrm{VCO}$ is amplified and injected into the SOA gain medium of the AKSS. This generates a narrow band laser output, having a linewidth $\delta \lambda$ and $f_{R}$ repetition rate. If $f_{S}=f_{R}$, no tuning is noticed. To perform tuning, sweeping needs to be performed at a detuned rate from $f_{R}$. This makes the regime used here different from that reported in FDML lasers [5].

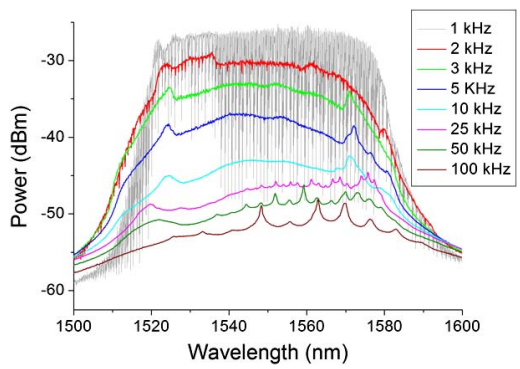

Fig. 3. Dynamic sweep optical output using the 1st modelocking mechanism only. 
Therefore, $f_{S}=f_{R} \pm \delta f_{R}$, in which case the VCO is tuned with a comb spectrum consisting in $f_{m}, f_{m}+f_{R} \pm \delta f_{R}$, $f_{m}+2 f_{R} \pm 2 \delta f_{R}, f_{m}+3 f_{R} \pm 3 \delta f_{R}$, and so on. The resulting RF signal from the VCO is amplified and injected into the SOA, therefore generating a comb laser output. The output bandwidth $\Delta \lambda$ is given by:

$$
\Delta \lambda=\frac{\Delta F}{f_{R}} C_{m} \cdot \delta f_{R} \cdot \delta \lambda,
$$

where $C_{m}$ is a cavity coefficient, and $\Delta F$ represents the frequency tuning range of the VCO.

Therefore, for the ring cavity length considered, the tuning bandwidth is measured for several values of detuning, $\delta f_{R}$. The results are presented in Fig. 4 . The larger the detuning, $\delta f_{R}$, less is the output power, as illustrated in Fig. 4 (left).

The cavity coefficient defined in Eq. (4) is evaluated from the slope $m=\delta \lambda \cdot N \cdot C_{m}$ calculated from the left graph illustrated in Fig. $\underline{4}$ (blue dashed line) as $18.72 \mathrm{~nm} / \mathrm{kHz}$.

Figure 4 (right) represents more explicitly the effects of the DML mechanism applied to the AKSS ring. When the frequency of the signal driving the VCO is swept at a rate $f_{S}=f_{R}=782 \mathrm{kHz}$, a narrow laser emission results. For a sweeping rate of $f_{S}=f_{R}+1=783 \mathrm{kHz}$, the VCO is tuned from $f_{m}$ to $f_{m}+f_{R}+1 \mathrm{kHz}$, to $f_{m}+$ $2 f_{R}+2 \mathrm{kHz}$, to $f_{m}+3 f_{R}+3 \mathrm{kHz}$, and so on, in the $880-940 \mathrm{MHz}$ range, in 783-kHz steps. The emitted spectrum consists in a tooth comb having a linewidth of $60 \mathrm{pm}$ and a repetition rate of $783 \mathrm{kHz}$. For a sweeping rate of $f_{S}=f_{R}+5=787 \mathrm{kHz}$, the $\mathrm{VCO}$ is tuned from $f_{m}$ to $f_{m}+f_{R}+5 \mathrm{kHz}$, to $f_{m}+2 f_{R}+10 \mathrm{kHz}$, to $f_{m}+3 f_{R}+$ $15 \mathrm{kHz}$, and so on, in the $880-940 \mathrm{MHz}$ range, in 787$\mathrm{kHz}$ steps. The emitted spectrum consists in a tooth comb having a linewidth of $60 \mathrm{pm}$ and a repetition rate of $787 \mathrm{kHz}$. Similar reasoning can be applied to each value of $\delta f_{R}$. The temporal output signal measured using a Newport 1611 1-GHz photodetector, for several values of detuning is represented in Fig. 5 . There is a slow decrease in power until $10-\mathrm{kHz}$ detuning. Using the procedure described for ortho-relative intensity noise (RIN) measurement described in [9], we have measured for 1,5 , and $10-\mathrm{kHz}$ detuning a RIN of $1.4 \%, 1.65 \%$, and $6.37 \%$, respectively.

For a mode-locking frequency of $920.4 \mathrm{MHz}$, at the sweeping rate of $792 \mathrm{KHz}\left(10 \mathrm{kHz}\right.$ detuning from $\left.f_{R}\right)$, we obtained a tuning bandwidth of $15.82 \mathrm{~nm}$, as shown
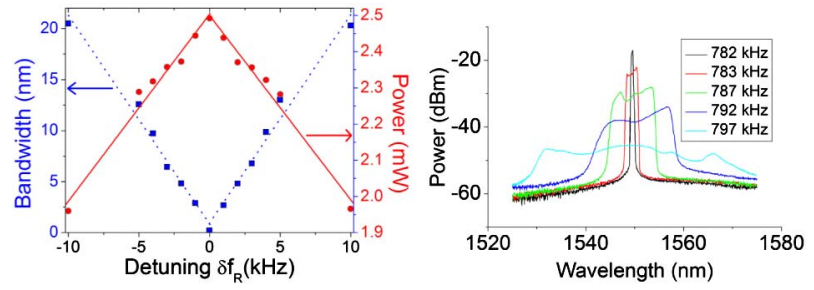

Fig. 4. (Left) DML AKSS tuning bandwidth (blue dotted line) and output power versus detuning (red solid line); (right) DML optical spectrum in the dynamic regime for a cavity length determining an $f_{R}=782 \mathrm{kHz}$, for detuning $\delta f_{R}=+1,+5,+10$, and $+15 \mathrm{kHz}$, respectively.

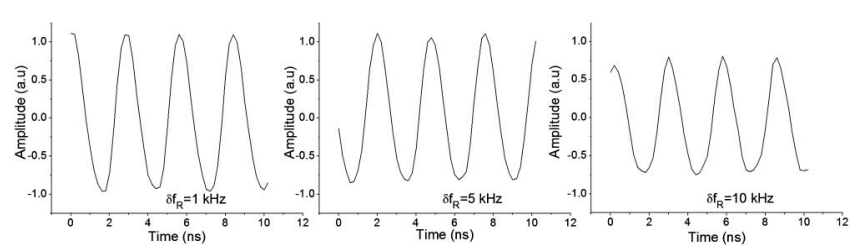

Fig. 5. Dynamic output measured for $\delta f_{R}=1 \mathrm{kHz}, 5 \mathrm{kHz}$, and respectively, $10 \mathrm{KHz}$.

in Fig. 4. Without any optical amplification, the output power of the source is $2.3 \mathrm{~mW}$. Using the SOA booster driven at $300 \mathrm{~mA}$, an output power of $12.8 \mathrm{~mW}$ is measured. The booster can deliver more power, however, a trade-off is established between output power and percentage of amplified spontaneous emission (ASE). While it is possible to tune the whole tuning bandwidth by $\pm 30 \mathrm{~nm}$ by modifying the bias voltage applied to the VCO, it is not possible to achieve the same tuning bandwidth dynamically. This is due to the maximum achievable VCO tuning bandwidth $\Delta F$ of $60 \mathrm{MHz}$ for a swing of maximum allowed of $10 \mathrm{~V}_{\mathrm{pp}}$ amplitude of the ramp signal applied to its input. A simple calculation, $60 \mathrm{~nm} / 15 \mathrm{~nm}=4$, shows that we would need a VCO with a 4-times-larger frequency swing to achieve the available tuning bandwidth of $30 \mathrm{~nm}$. The dependence of the optical output power and tuning bandwidth on the $\mathrm{RF}$ tuning range is depicted in Fig. 6 , for two values of detuning, 5 and $10 \mathrm{KHz}$. The variation of tuning bandwidth with amplitude represents an expansion of the number of frequency resolved points in the tuned spectrum. For the same $\delta f_{R}$ detuning value, there is a slight decrease in power as the VCO tuning range $\Delta F$ increases, while the bandwidth exhibits a significant increase. The graphs depicted in Fig. 6 confirm Eq. (4). For constant $f_{R}$, $C_{m}$, and $\delta \lambda$ values, the tuning bandwidth is proportional to the detuning $\delta f_{R}$. For example, for a 5 -Vpp signal driving the $\mathrm{VCO}$, corresponding to $\Delta F=30 \mathrm{MHz}$, a $\Delta \lambda$ value of $7.86 \mathrm{~nm}$ is measured for $\delta f_{R}=5 \mathrm{kHz}$, and respectively, $15.24 \mathrm{~nm}$ at $10 \mathrm{kHz}$. This discussion makes distinction between the conventional drive of a dispersive cavity with a VCO tuned at a low frequency and the DML regimes described here. In the first case, the RF frequency of the VCO is tuned within one locking band. In the DML regime, the VCO is tuned over many such locking bands, separated by $f_{R}$.

The DML tuning mechanism is also verified at multiple values of $f_{R}$. Equation (4) suggests that the tuning bandwidth, $\Delta \lambda$, is inversely proportional to $f_{R}$. For a
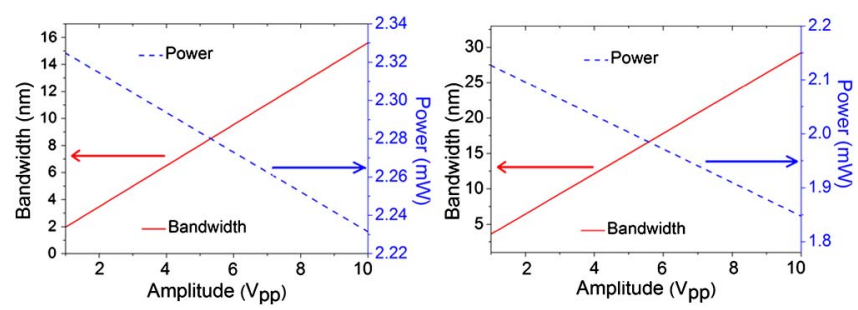

Fig. 6. AKSS optical output power (dashed blue line) and tuning bandwidth (solid red line) versus the amplitude of the ramp applied to the VCO input for a detuning of $5 \mathrm{kHz}$ (left), respectively, $10 \mathrm{kHz}$ (right). 


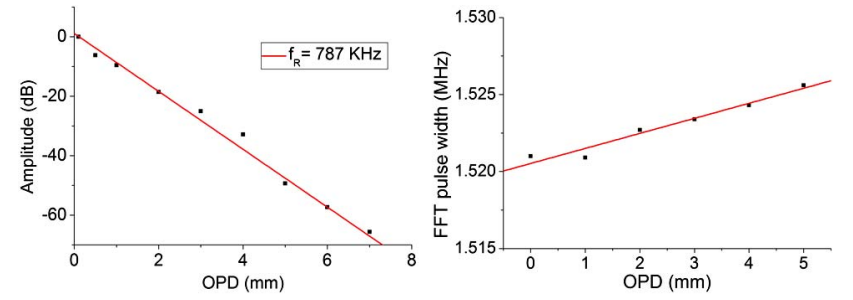

Fig. 7. (left) Decay of modulation intensity with increasing OPD; (right) A-scan pulse width versus OPD.

5.3-Vpp signal driving the VCO, a $15.75-\mathrm{nm} \Delta \lambda$ value is measured for a $\delta f_{R}=10 \mathrm{kHz}$ detuning from $f_{R}$. For the same detuning, by modulating the VCO at sweeping rates of $2 f_{R}(1.564 \mathrm{MHz}), 3 f_{R}(2.346 \mathrm{MHz}), 4 f_{R}(3.128 \mathrm{MHz})$, and $5 f_{R}(3.910 \mathrm{MHz})$ we measured $\Delta \lambda$ as $7.9 \mathrm{~nm}$, $5.5 \mathrm{~nm}, 4.2 \mathrm{~nm}$, and, respectively, $3.2 \mathrm{~nm}$, fact that confirms the theoretical hypothesis. The proportional reduction in the tuning bandwidth is accompanied by insignificant power variation.

The spectrum of the RF signal of a carrier $f_{m}$ when swept in frequency at a rate $f_{s}$ consists in a comb of frequencies separated by $f_{s}$. The number of resolved frequency points within the spectrum, $S$, is given by the maximum frequency deviation, $\Delta F$, divided by $f_{S}$. $\Delta F$ depends on the voltage applied to the tuning input of the synthesizer, when the switch in Fig. 1 is on A or to the input of the VCO when the switch is on B. If $f_{s}=1 \mathrm{kHz}$, as $\Delta F$ is less than $f_{R}=782 \mathrm{kHz}$, maximum achievable for $S$ would be 782. When driving the AKSS at a much larger frequency $f_{R}$, the only way to maintain a large number of resolved frequency points is to jump the optical frequency of the swept source from one tuning band to the next, in steps of $f_{R}$. In the DML regime, a deviation of frequency as high as $S \cdot f_{R}=782 \cdot 782=$ $611.52 \mathrm{MHz}$ would therefore be needed.

Finally, we evaluated the dynamic linewidth $\delta \lambda$ of the AKSS, as the inverse of this parameter determines the axial range in OCT. A 255-m-length AKSS cavity, mode locked at $920.7 \mathrm{MHz}$, swept in frequency at around $787 \mathrm{kHz}$ and using the maximum $\Delta F=60 \mathrm{MHz}$ deviation achievable by driving the $\mathrm{VCO}$ at $10 \mathrm{~V}$, is connected to a Mach-Zehnder interferometer. By applying a fast Fourier transformation (FFT) oscilloscope function on the output signal measured by the detector and displayed on the oscilloscope, for various optical path difference (OPD) values, we have measured the FFT peaks and obtained the modulation intensity versus OPD for each configuration, as depicted in Fig. $\underline{6}$ (left). The sensitivity is given by $20 \cdot \log \left(A_{\mathrm{OPD}} / A_{\text {noise }}\right)$, where $A_{\mathrm{OPD}}$ represents the corresponding FFT peak amplitude value for the chosen OPD, while $A_{\text {noise }}$ is the amplitude of the noise floor measured outside the FFT peak [10]. A sensitivity value of $91.12 \mathrm{~dB}$ is obtained for an $\overline{\mathrm{OPD}}$ value close to " 0 ". Assuming a Gaussian shape for the tuning spectrum, and using the $\mathrm{OPD}_{6 \mathrm{~dB}}$ from Fig. $\underline{6}$ (left) of $0.75 \mathrm{~mm}$, this leads to a dynamic linewidth $\delta \lambda=\lambda^{2} / 4 \cdot \mathrm{OPD}_{6 \mathrm{~dB}}=$ $0.8 \mathrm{~nm}$. In terms of tuning linearity, the width of the A-scan peaks obtained by FFT has shown insignificant change with the OPD, which represents an increase of only $0.4 \%$ at $\mathrm{OPD}=5 \mathrm{~mm}$, in comparison with the width of the A-scan peak at an OPD close to zero, as represented in Fig. 7 (right). This suggests sufficient tuning linearity in optical frequency, i.e., no data resampling is needed when decoding the spectrum modulation.

Using the DML principle, operation at fast sweep rate values of $f_{R}=782 \mathrm{kHz}$ are demonstrated, and also up to 5 multiples of $f_{R}$, with a proportional decrease in the tuning bandwidth. Further optimization of the RF tuning can lead to larger tuning bandwidths and better sensitivity. The DML mechanism allows $\mathrm{MHz}$ rates to be obtained directly, with no buffering [11], reducing the cost and complexity in producing a swept source.

The work was supported by the European Research Council under the European Union's Seventh Framework Program, Advanced Grant agreement "COGATIMABIO”, No. 249889. A. Podoleanu is also supported by the NIHR Biomedical Research Center at Moorfields Eye Hospital NHS Foundation Trust and UCL Institute of Ophthalmology.

\section{References}

1. S. H. Yun, G. Boudoux, G. J. Tearney, and B. E. Bouma, Opt. Lett. 28, 1981 (2003).

2. I. Trifanov, A. Bradu, L. Neagu, P. Guerreiro, A. B. L. Ribeiro, and A. G. Podoleanu, IEEE Photon. Technol. Lett. 23, 825 (2011).

3. J. Masson, R. St-Gelais, A. Poulin, and Y.-A. Peter, IEEE J. Quantum Electron. 46, 1313 (2010).

4. T.-H. Tsai, O. O. Ahsen, H.-C. Lee, K. Liang, M. G. Giacomelli, B. Potsaid, Y. K. Tao, V. Jayaraman, M. F. Kraus, J. Hornegger, M. Figueiredo, Q. Huang, H. Mashimo, A. E. Cable, and J. G. Fujimoto, Proc. SPIE 8927, 89270T (2014).

5. R. H. Huber, M. Wojtkowski, and J. G. Fujimoto, Opt. Express 14, 3225 (2006).

6. S. Yamashita, Y. Nakazaki, R. Konishi, and O. Kusakari, J. Comp. Physiol. A 2009, 572835 (2009).

7. Y. Nakazaki and S. Yamashita, Opt. Express 17, 8310 (2009).

8. Y. Takubo and S. Yamashita, Opt. Express 21, 5130 (2013).

9. B. R. Biedermann, W. Wieser, C. M. Eigenwillig, T. Klein, and R. Huber, Opt. Express 17, 9947 (2009).

10. A. G. Podoleanu and A. Bradu, Opt. Express 21, 19324 (2013).

11. R. Huber, D. C. Adler, and J. G. Fujimoto, Opt. Lett. 31, 2975 (2006). 